\title{
Intraductal papillary mucinous neoplasms of the pancreas: Clinical association with KRAS
}

\author{
XIAO YAN CHANG *, YAN WU*, YUAN LI, JING WANG and JIE CHEN \\ Department of Pathology, Peking Union Medical College Hospital, \\ Chinese Academy of Medical Sciences and Peking Union Medical College, Tsinghua University, Beijing 100730, P.R. China
}

Received May 25, 2017; Accepted January 18, 2018

DOI: $10.3892 / \mathrm{mmr} .2018 .8875$

\begin{abstract}
Intraductal papillary mucinous neoplasms of the pancreas (IPMN) are among the most important precancerous lesions in the pancreas. V-Ki-ras 2 Kirsten rat sarcoma viral oncogene homolog (KRAS) is one of the most important genes involved in pancreatic neoplasms, and exhibits a high mutation rate in pancreatic ductal adenocarcinomas and pancreatic intraepithelial neoplasia. The present study aimed to further elucidate the associations among IPMN subtypes (gastric, intestinal, pancreatobiliary and oncocytic), pathological classifications [low-grade, intermediate-grade, and high-grade IPMN, and associated minimally invasive carcinoma (invasive depth $\leq 0.5 \mathrm{~cm}$ ) and advanced invasive carcinoma (invasive depth $>0.5 \mathrm{~cm}$ )]. A total of 56 cases of IPMN were studied using scorpion amplified refractory mutation system analysis of KRAS mutations, pathological features and prognosis. KRAS mutations were identified in 50\% (28/56 cases). The frequency was $60 \%$ (9/15 cases) in gastric-type, $52.6 \%$ (10/19 cases) in intestinal-type, $47.3 \%$ (9/19 cases) in pancreatobiliary-type and zero (0/3 cases) in oncocytic-type IPMN. Except for oncocytic type IPMN, the frequencies of KRAS mutations in IPMN with low, intermediate and high grade, and IPMN-associated
\end{abstract}

Correspondence to: Dr Jie Chen, Department of Pathology, Peking Union Medical College Hospital, Chinese Academy of Medical Sciences and Peking Union Medical College, Tsinghua University, 1 Shuaifuyuan Wangfujing, Beijing 100730, P.R. China

E-mail:xhblk@163.com

*Contributed equally

Abbreviations: IPMN, intraductal papillary mucinous neoplasms of the pancreas; IPMN-MI, IPMN with an associated micro-invasive carcinoma; IPMN-AI, IPMN with an associated advanced invasive carcinoma; IPMN-GT, gastric type IPMN; IPMN-IT, intestinal type IPMN; IPMN-PT, pancreatobiliary type IPMN; PDA, pancreatic ductal adenocarcinomas; KRAS, V-Ki-ras 2 Kirsten rat sarcoma viral oncogene homolog; ABI, Applied Biosystems; ARMS, amplified refractory mutation system; qPCR, quantitative polymerase chain reaction

Key words: IPMN, pancreas, KRAS mutation, survival carcinoma were $58.3 \%$ (7/12 cases), $27.3 \%$ (3/11 cases), $80 \%$ ( $4 / 5$ cases) and $56 \%$ (14/25 cases), respectively. With more advanced dysplasia and invasion, the prevalence of KRAS mutations in intestinal-type IPMN increased $(\mathrm{P}=0.012)$. The Kaplan-Meier survival curve demonstrated that survival rate was not associated with KRAS mutation (log-rank test; $\mathrm{P}=0.308$ ). The prevalence of KRAS mutations was lowest in intestinal-type IPMN, and was in proportion to the degree of dysplasia and invasion. Therefore, KRAS mutation in IPMN does not correlate with histological subtype, dysplasia grade, depth of invasion or survival.

\section{Introduction}

Intraductal papillary mucinous neoplasms of the pancreas (IPMN) are one of the most important precancerous lesions associated with pancreatic cancers. IPMN are slow-growing intraductal tumors with a favorable prognosis (1). With the development of radiological technology, intraductal lesions of the pancreas have been diagnosed increasingly frequently.IPMN currently accounts for $8-20 \%$ of all resected pancreatectomy specimens (2-6), and the incidence is increasing.

IPMN are characterized by intraductal papillary proliferation of mucin-producing cells, which causes cystic dilation of the pancreatic ducts. IPMN are different from pancreatic intraepithelial neoplasias (PanINs). IPMN are visible and detectable lesions with a diameter $>1 \mathrm{~cm}$, whereas PanINs are diagnosed microscopically and are usually $<0.5 \mathrm{~cm}$ in diameter (7). Similar to the adenoma-carcinoma sequence that is observed in colon cancer, IPMN stand to progress from adenomatous lesions (IPMN with low- or intermediate- grade dysplasia) to high-grade dysplasia and finally to invasive carcinomas (IPMN-associated carcinomas), which exhibit the most severe architectural and cytological atypia. Histologically, IPMN may be divided into gastric-type, intestinal-type, pancreatobiliary-type and oncocytic-type, according to the epithelial lining. Each histological type has specific histopathological features and biological behaviors. IPMN-associated carcinomas primarily comprise pancreatic ductal adenocarcinomas (PDAs) and colloid carcinomas. It has been previously demonstrated that intestinal-type IPMNs usually progress to pancreatic carcinomas via a colloidal pattern, while pancreatobiliary-type progress via a tubular pattern. Minimally invasive IPMN is defined by 
Nara et al (8) as a lesion that invades slightly beyond the ductal wall.

KRAS, a member of the ras gene family, is one of the most important and commonly mutated oncogenes observed in pancreatic neoplasms (9). Numerous reports have confirmed that KRAS mutations serve a dominant and important role in the tumorigenesis of PDAs and IPMN (10-14). Previous studies have discussed the association between KRAS mutations and survival in patients with PDAs, and demonstrated that mutations were not associated with poor prognosis (15-18). Previous studies have reported that point mutations are located primarily at codon 12 in exon 2 in $31-86 \%$ of patients with IPMN (10-14,19-22). There have been few studies elaborating upon the alteration of KRAS mutation frequency among different subtypes (gastric, intestinal or pancreatobiliary type) or different grades (low, intermediate and high grade). Few articles have discussed the correlation between prognosis and KRAS mutation status in patients with IPMN (23).

In China, to the best of our knowledge, there is no available research regarding the incidence of IPMN or other associated data. A total of 56 IPMN cases were collected for the present study, clinicopathological analysis was performed and KRAS mutation status was detected. The objective of the present study was to discuss the clinical meaning of KRAS mutations and their association with histological classification, and subtypes, invasion and survival of IPMN.

\section{Materials and methods}

Study population. All of the pancreatic specimens were obtained from Peking Union Medical College Hospital, Beijing, China. Between January 2000 and December 2009, a total of 900 pancreatic tumors were resected and confirmed by pathology analysis. A total of two expert pathologists reviewed all slides and classified the cases according to the new World Health Organization (WHO) classification system. A total of 61 patients were diagnosed with IPMN, and 29 exhibited invasive carcinomas associated with IPMN. Of the 61 IPMN cases, there was a failure to extract sufficient genomic DNA to assess KRAS genetic alterations in five cases. All IPMN lesions were associated with the pancreatic duct system, and microscopic observation did not reveal any 'ovarian-type' hypercellular stroma surrounding the neoplasms. The present study was approved by the Ethics Committee of Peking Union Medical College Hospital, and informed consent was obtained from all cases.

Pathological examination of IPMN. Histological grading of the IPMN was performed according to the criteria defined by the 2010 edition of the WHO classification system for digestive neoplasms (24). Infiltrative growth accompanied by desmoplastic fibrosis was used as the only criterion to determine infiltration. A small number of samples exhibited evidence of mucin leaking into the interstitial space and forming a simple mucous lake around the pancreatic duct in the absence of floating cancer cells, and this was not regarded as invasion in the present study. Invasive depth was measured from the duct to the deepest infiltrating cancer cells. A cut-off point of $0.5 \mathrm{~cm}$ was selected for the depth of invasion and the cases were divided into two groups: Cases with an invasive depth $\leq 0.5 \mathrm{~cm}$ was designated as IPMN-associated minimally invasive carcinoma (IPMN-MI), and invasive depth $>0.5 \mathrm{~cm}$ was designated as IPMN-associated advanced invasive carcinoma (IPMN-AI). All sections were observed at a magnification of x40, 100 and 200 by Olympus BX53 microscope (Olympus Corporation, Tokyo, Japan).

Mutation analysis. Neutral $10 \%$ formalin-fixed (at room temperature, overnight) and paraffin-embedded pancreatic neoplasm tissue sections were cut $(6 \mu \mathrm{m})$, and subsequently microdissected manually to ensure the highest purity and the largest amount of tumor cells in each sample. The resection margins with non-tumorous tissue served as normal controls. Genomic DNA was extracted using the QIAmp DNA Mini kit (Qiagen Inc., Valencia, CA, USA).

A DxS ARMS KRAS mutation test kit (DXS International PLC, Farnham, UK) was used to detect point mutations in codons 12 and 13 of exon 2 of the KRAS gene. This ARMS assay was a quantitative polymerase chain reaction (qPCR) assay that detected a total of eight genetic mutations, including $35 \mathrm{G}>\mathrm{A}, 35 \mathrm{G}>\mathrm{T}, 35 \mathrm{G}>\mathrm{C}, 34 \mathrm{G}>\mathrm{A}, 34 \mathrm{G}>\mathrm{T}, 34 \mathrm{G}>\mathrm{C}, 37 \mathrm{G}>\mathrm{C}$ and $38 \mathrm{G}>\mathrm{A}$. The mutation test was performed according to the manufacturer's protocol. qPCR was performed using an internal Scorpion reporter probe and primers specific for wild and mutant KRAS on the ABI 7500 Real-time polymerase chain reaction system (ABI; Thermo Fisher Scientific, Inc., Waltham, MA, USA). The following thermocycling conditions were used for the qPCR: $50^{\circ} \mathrm{C}$ for $2 \mathrm{~min}$, initial denaturation at $95^{\circ} \mathrm{C}$ for $10 \mathrm{~min} ; 40$ cycles of $95^{\circ} \mathrm{C}$ for $15 \mathrm{sec}$ and $60^{\circ} \mathrm{C}$ for $10 \mathrm{sec}$. Data analysis was performed using the ABI SDS software v1.4.0.25 (Thermo Fisher Scientific, Inc.). The differences between the cycle threshold (CT) values of the mutant and wild types were calculated. When the difference of CT value between wild and mutant type was less than the cut-off value, we determined that there was a corresponding mutation in the sample $(25,26)$. Subsequently, the mutation of KRAS in codon 61 was detected via direct sequencing of the PCR amplification product with an automated ABI 3730 sequencer (ABI; Thermo Fisher Scientific, Inc.). The primers for codon 61 of KRAS were 5'-TCCCTTCTCAGGATTCCT ACA-3' and 5'-CAAAGAAAGCCCTCCCCAGT-3'. The following thermocycling conditions were used for the PCR amplification of codon 61: Initial denaturation at $94^{\circ} \mathrm{C}$ for $5 \mathrm{~min} ; 40$ cycles of $94^{\circ} \mathrm{C}$ for $30 \mathrm{sec}, 58^{\circ} \mathrm{C}$ for $30 \mathrm{sec}$ and $72^{\circ} \mathrm{C}$ for $30 \mathrm{sec}$; and final extension for $10 \mathrm{~min}$ at $72^{\circ} \mathrm{C}$. The amplification was performed using TaKaRa Ex Taq polymerase (Takara Bio Inc., Otsu, Japan).

Statistical analysis. All statistical analyses were performed using SPSS version 12.0 (SPSS Inc., Chicago, IL, USA) software. Data are presented as the mean value. $\mathrm{P}<0.05$ was considered to indicate a statistically significant difference. The comparison between continuous variables was calculated using a Student's t-test. The correlation between categorical variables was acquired by $\chi^{2}$ test or Fisher's exact test. The $\chi^{2}$ test was used in the IPMN constituent ratio analysis. The Kaplan-Meier method was used to assess survival time, and the log-rank test was applied to determine significance. 
Table I. Association between KRAS mutation and the clinicopathological features of IPMN.

\begin{tabular}{|c|c|c|c|}
\hline Feature & KRAS+ $(\%)$ & KRAS- $(\%)$ & P-value \\
\hline Sex & & & $0.408^{\mathrm{a}}$ \\
\hline Male & $19(76.0)$ & $16(64.0)$ & \\
\hline Female & $9(42.9)$ & $12(57.1)$ & \\
\hline Age, years & & & $>0.9^{\mathrm{a}}$ \\
\hline$\geq 65$ & $12(50.0)$ & $12(50.0)$ & \\
\hline$<65$ & $16(50.0)$ & $16(50.0)$ & \\
\hline Mean size, $\mathrm{cm}$ & 3.50 & 3.71 & $0.718^{\mathrm{b}}$ \\
\hline Pancreatitis & & & $0.408^{\mathrm{a}}$ \\
\hline Yes & $12(57.1)$ & $9(42.9)$ & \\
\hline No & $16(64.0)$ & $19(76.0)$ & \\
\hline Smoking & & & $0.771^{\mathrm{a}}$ \\
\hline Yes & $9(52.9)$ & $8(47.1)$ & \\
\hline No & $19(48.7)$ & $20(51.3)$ & \\
\hline Macroscopic type & & & $0.695^{\mathrm{c}}$ \\
\hline Main duct type & $14(45.2)$ & $17(54.8)$ & \\
\hline Branch duct type & $4(50.0)$ & $4(50.0)$ & \\
\hline Mixed type & $10(58.8)$ & $7(41.2)$ & \\
\hline Histological classification & & & $0.605^{\mathrm{a}}$ \\
\hline Low grade & $7(58.3)$ & $5(41.7)$ & \\
\hline Intermediate grade & $3(27.3)$ & $8(72.7)$ & \\
\hline High grade & $4(57.1)$ & $3(42.9)$ & \\
\hline With associated carcinoma & $14(53.8)$ & $12(46.2)$ & \\
\hline Histological subtype & & & $0.159^{c}$ \\
\hline Gastric-type & $9(60.0)$ & $6(40.0)$ & \\
\hline Intestinal-type & $10(52.6)$ & $9(47.4)$ & \\
\hline Pancreatobiliary-type & $9(47.4)$ & $10(52.6)$ & \\
\hline Oncocytic-type & $0(0)$ & $3(100)$ & \\
\hline IPMN/associated carcinoma & & & $0.592^{\mathrm{a}}$ \\
\hline Yes & $14(53.8)$ & $12(46.2)$ & \\
\hline No & $14(46.7)$ & $16(53.3)$ & \\
\hline
\end{tabular}

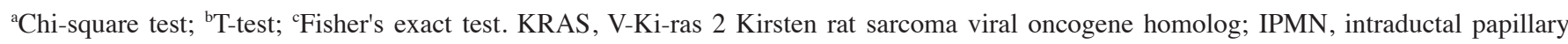
mucinous neoplasms of the pancreas.

\section{Results}

IPMNs subtypes and classification. Of the 56 cases of IPMN, 33 were in the head of the pancreas, 12 in the tail of the pancreas, and 11 in the whole pancreas. The age of patients ranged between 39 and 79 years, and the average was 61.8 years. The onset manifestation was abdominal discomfort (abdominal pain, abdominal distention and diarrhea) in 34 cases. A total of 13 cases were detected accidentally with no clinical symptoms. The remaining 9 cases represented symptoms with jaundice $(n=4)$, weight loss $(n=2)$, back pain $(n=1)$, a high level of cancer antigen $199(n=1)$ and a high creatinine level $(n=1)$. Sex, age and tumor location were not associated with invasion. However, 21/56 (37.5\%) patients had a history of acute or chronic pancreatitis with a high amylase level in the blood on at least one occasion prior to the surgery, and 17 patients smoked for $>10$ years. However, pancreatitis
$(\mathrm{P}=0.408)$ and smoking $(\mathrm{P}=0.771)$ were not associated with KRAS mutations.

IPMN were subclassified into four histological subtypes: gastric-type $(15 / 56 ; 26.8 \%)$, intestinal-type $(19 / 56 ; 33.9 \%)$, pancreatobiliary-type $(19 / 56 ; 33.9 \%)$ and oncocytic-type (3/56; 5.3\%). Based on the highest degree of architectural and cytological atypia, the present cohort comprised $12(21.4 \%)$ IPMN cases with low-grade dysplasia, 11 (19.6\%) IPMN cases with intermediate-grade dysplasia, 7 (12.5\%) IPMN cases with high-grade dysplasia and 26 (46.4\%) IPMN-associated invasive carcinomas (TableI). The majority of the gastric-type IPMN cases (14/15) were classified as IPMN with low- and intermediate-grade dysplasia (Fig. 1). Almost all of the pancreatobiliary-type IPMN cases (18/19) were IPMN-associated carcinomas. This suggested that pancreatobiliary-type IPMN are significantly associated with high grade dysplasia and invasive adenocarcinoma, while gastric-type IPMN were associated with low and intermediate 


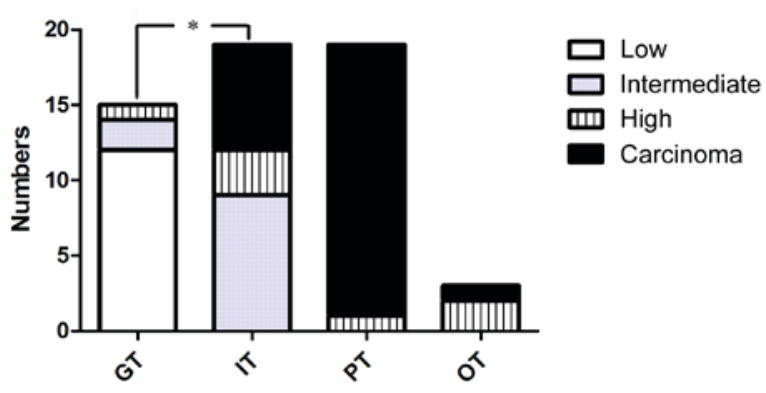

Figure 1. Numbers and ratio of IPMN with low-, intermediate- and high-grade dysplasia and IPMN with an associated carcinoma in GT, IT, PT and OT IPMN. PT was associated with high grade dysplasia of IPMN and invasion; in addition, GT IPMN was associated with low and intermediate grade dysplasia. "P<0.05. IPMN, intraductal papillary mucinous neoplasms of the pancreas; GT, gastric-type; IT, intestinal-type; PT, pancreatobiliary-type; OT, oncocytic-type.

grade dysplasia ( $\mathrm{P}=0.004)$. IPMN-associated carcinomas were primarily PDAs $(21 / 26 ; 80.8 \%)$, colloid carcinomas $(4 / 26 ; 15.4 \%)$ and, rarely, undifferentiated carcinomas $(1 / 26 ; 3.8 \%)$. A total of 11 cases belonged to the IPMN-microinvasive group, including 10 tubular adenocarcinomas and one colloid carcinoma.

Survival of patients with IPMNs. A total of five out of 56 patients were lost to follow-up, 35 patients were alive and tumor-free, four patients were alive with IPMN, and 12 patients succumbed to IPMN-associated invasive carcinoma. The mean postoperative follow-up period was 40.8 months (range, 12-112 months) for living patients. The overall 5-year survival rate was $76 \%$. The 5-year survival rate of the patients with non-invasive IPMN was $100 \%$. The 5 -year survival rate of the patients with invasive IPMN was not available; instead, the 3-year survival rate of the patients with invasive IPMN was 55\%. Of the 12 patients who succumbed, eight presented with pancreatobiliary-type IPMN, three had intestinal-type IPMN and one had oncocytic-type IPMN. A patient diagnosed with colloid carcinoma succumbed due to a complication of Trousseau syndrome 11 months post-surgery. Of the 12 patients who succumbed, all of them presented with IPMN-associated carcinomas and succumbed 7-41 months post-surgery; 11 of these patients succumbed within 2 years. A total of four patients developed recurrent tumors in the remnant pancreas, and these patients presented with gastric-type IPMN with low-grade dysplasia, intestinal-type IPMN with intermediate-grade dysplasia, and intestinal-type and oncocytic-type IPMN with high-grade dysplasia, respectively. Recurrent tumors remained within the pancreatic ducts and exhibited the same histological features as their origin tumors. The first two patients had positive surgical margins in the first segmental pancreatectomy operation.

Disease-specific survival rates were analyzed using the Kaplan-Meier method. Upon comparison of the survival stages of IPMN-MI and IPMN-AI, it was identified that the survival stage of IPMN-AI tumors was significantly shorter compared with that of IPMN-MI tumors (log-rank test; $\mathrm{P}=0.0006$; Fig. 2A). Among the three histological subtypes, gastric-type IPMN had the best prognosis, pancreatobiliary-type IPMN had the worst, and intestinal-type IPMN lay in between (log-rank test; $\mathrm{P}<0.0001$; Fig. 2B).
A

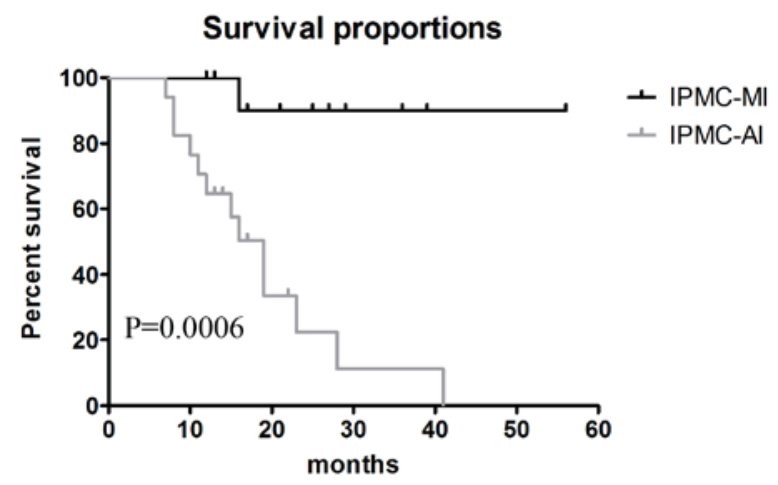

B

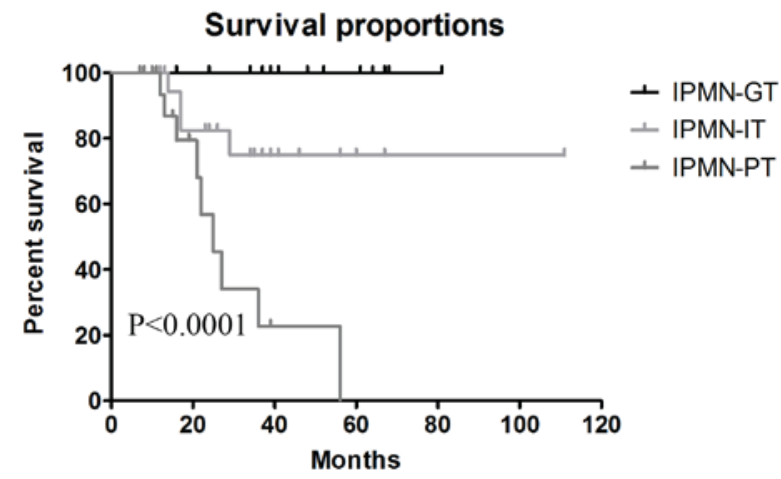

C

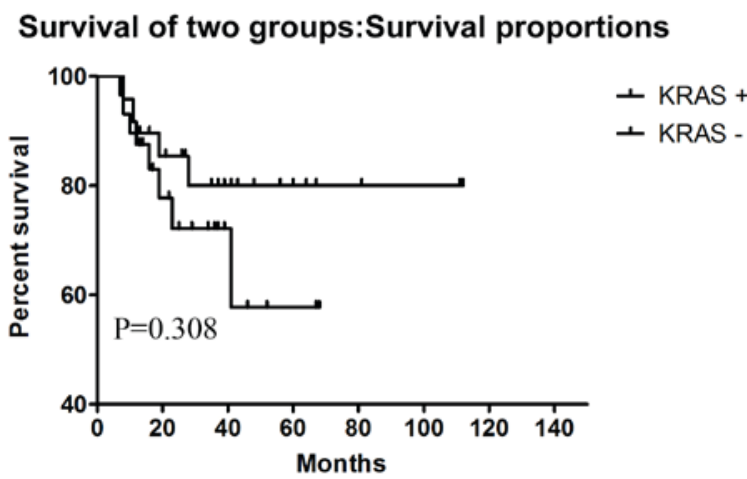

Figure 2. Kaplan-Meier survival curves of 56 patients with IPMN. (A) Overall survival following resection of IPMN-MI was significantly improved compared with IPMN-AI. (B) The overall survival of patients with GT-IPMN was increased compared with patients with IT-IPMN, while the overall survival of patients with PT-IPMN was the worst. (C) The overall survival of patients with KRAS mutations exhibited no marked difference compared with the KRAS non-mutated population. IPMN, intraductal papillary mucinous neoplasms of the pancreas; IPMN-MI, IPMN-associated minimally invasive carcinoma; IPMN-AI, IPMN-associated advanced invasive carcinoma; GT, gastric-type; IT, intestinal-type; PT, pancreatobiliary-type; KRAS, V-Ki-ras 2 Kirsten rat sarcoma viral oncogene homolog.

Pancreatobiliary-type IPMN were more likely to develop into infiltrative carcinomas.

KRAS mutations. KRAS mutations were identified in 28 of the 56 tested patients, with a rate of $50 \%$. No mutation was detected in the matching normal tissues. The frequency of KRAS mutations was $60 \%$ (9/15 cases) in gastric-type IPMN, $52.6 \%$ (10/19 cases) in intestinal-type, and 47.3\% (9/19 cases) in pancreatobiliary-type. KRAS mutations were not present in oncocytic-type IPMN. Except for oncocytic-type IPMN, 
Table II. V-Ki-ras 2 Kirsten rat sarcoma viral oncogene homolog mutational ratio of gastric-type, intestinal-type and pancreatobiliary-type IPMN in IPMN with low and intermediate grade dysplasia, high grade dysplasia, and associated carcinoma.

\begin{tabular}{lccc}
\hline & Gastric-type $(\mathrm{n}=15)$ & Intestinal-type $(\mathrm{n}=19)$ & Pancreatobiliary-type $(\mathrm{n}=19)$ \\
\hline Low and intermediate grade & $8 / 14(57.1 \%)$ & $2 / 9(22.2 \%)$ & 0 \\
High grade & $1 / 1$ & $2 / 3$ & $1 / 1$ \\
Associated carcinoma & 0 & $6 / 7(85.7 \%)$ & $8 / 18(44.4 \%)$ \\
\hline
\end{tabular}

IPMN, intraductal papillary mucinous neoplasms of the pancreas.

the frequencies of KRAS mutations in IPMN with low-, intermediate- and high-grade dysplasia, and IPMN-associated carcinomas were $58.3 \%$ ( $7 / 12$ cases), $27.3 \%$ (3/11 cases), $80 \%$ (4/5 cases) and 56\% (14/25 cases), respectively. KRAS mutation rates in low-grade $(58.3 \%)$ and high-grade IPMNs $(80 \%)$ were increased compared with intermediate-grade IPMN (27\%). There was no association between KRAS mutations and histological subtype $(\mathrm{P}=0.159)$, or invasive cancer $(\mathrm{P}=0.592)$ (Table I).

There were certain differences in KRAS mutations among the three histological subtypes. Of the 19 intestinal-type IPMN cases, 6/7 invasive intraductal papillary mucinous carcinoma exhibited KRAS mutations, and 2/9 borderline IPMN exhibited KRAS mutations. With the increased degree of dysplasia and invasion of intestinal-type IPMN, KRAS mutation rates increased $(\mathrm{P}=0.012)$ (Table II). Compared with intestinal-type IPMN with low- and intermediate-grade dysplasia, gastric-type IPMN with low- and intermediate-grade dysplasia had increased KRAS mutations (57.1 and 22.2\%). Intestinal-type IPMN with associated carcinomas had a higher KRAS mutation prevalence compared with pancreatobiliary-type IPMN with associated carcinomas (86.7 and 44.4\%). Due to the small number of cases, it was not possible to calculate a P-value.

The present study compared the histopathological features of the invasive group (26 cases) with KRAS mutation occurrence, and demonstrated that the infiltrative depth $(\mathrm{P}=0.753)$, differentiation $(\mathrm{P}=0.909)$, lymph node metastasis $(\mathrm{P}=0.177)$, infiltrative extent $(\mathrm{P}=0.419)$ and the type of carcinoma $(\mathrm{P}=0.468)$ had no association with KRAS mutation occurrence (Table III). The Kaplan-Meier survival curve demonstrated that survival was not associated with KRAS mutation (log-rank test; $\mathrm{P}=0.308$; Fig. 2C).

The mutated site exhibited single-amino-acid substitutions, including G12D (13 cases; 41\%), G12V (12 cases; 38\%), G12S (two cases), G12A (one case), G12C (two cases), G12R (one case) and $61 \mathrm{CAA}>\mathrm{CGA}$ (one case), and double-amino-acid substitutions, including G12V/G12S (two cases), G12D/G12S (one case) and G12D/G12V (one case). Among the four cases with double mutations, three were invasive carcinomas and the other was a benign tumor. A total of eight out of 13 cases with G12D mutation were IPMN with high-grade dysplasia and/or IPMN-associated carcinomas, and seven out of 12 cases with G12V mutation were IPMN with high-grade dysplasia and/or IPMN-associated carcinomas. The altered amino acids were not associated with IPMN classification. No mutations were identified within codon 13 of exon 2.
Table III. Association between KRAS mutation, and pathological features and prognosis of intraductal papillary mucinous neoplasms of the pancreas-associated carcinoma.

\begin{tabular}{lccc}
\hline & KRAS+ & KRAS- & P-value \\
\hline $\begin{array}{l}\text { Infiltrative depth, cm } \\
\leq 0.5 />0.5\end{array}$ & $4 / 10$ & $7 / 5$ & $0.753^{\mathrm{a}}$ \\
Differentiation & & & \\
$\quad$ Well-differentiated & 7 & 7 & \\
$\quad$ Moderately-differentiated & 4 & 3 & \\
Poorly-differentiated & 3 & 2 & $0.909^{\mathrm{a}}$ \\
Lymph node metastasis & & & \\
$\quad$ Yes/no & $4 / 10$ & $2 / 10$ & $0.470^{\mathrm{a}}$ \\
Infiltrative scope & & & \\
Limited/adipose tissue & $8 / 6$ & $8 / 4$ & $0.619^{\mathrm{a}}$ \\
AJCC & & & \\
Stage I & 6 & 5 & \\
Stage II & 7 & 7 & \\
Stage III & 1 & 0 & \\
Stage IV & 0 & 0 & N/A \\
Carcinoma types & & & \\
Tubular/non-tubular & $10 / 4$ & $10 / 2$ & $0.468^{\mathrm{a}}$ \\
\end{tabular}

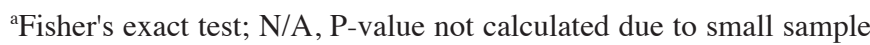
size. KRAS, V-Ki-ras 2 Kirsten rat sarcoma viral oncogene homolog; AJCC, American Joint Committee on Cancer.

\section{Discussion}

IPMN are a common cystic neoplasm located in the main and/or branch ducts of the pancreas. The histological features and classification of IPMN have been well characterized (2,27-32). The results of the present study indicated that histological subtype may suggest prognosis, similar to the reports by Mino-Kenudson et al (33), Furukawa et al (34) and Kang et al (35). Gastric-type IPMN have an overall favorable prognosis, exhibiting a very low risk of developing into carcinomas and with a longer survival compared with other subtypes (33-35). Almost all IPMN-associated colloid carcinomas progress from intestinal-type IPMN (36), which have a better prognosis compared with IPMN-associated tubular adenocarcinomas $(37,38)$. Pancreatobiliary-type 
IPMN frequently appear with high-grade dysplasia and usually progress into tubular adenocarcinomas (39). A total of 18 of the 19 pancreatobiliary-type IPMN were invasive IPMN in the present study. Furthermore, the incidence rates of the pancreatobiliary-type and intestinal-type lesions were equal, and tubular adenocarcinoma was the predominant type of neoplasm, rather than colloid carcinoma. Therefore, pancreatobiliary-type IPMN have the worst prognosis.

In accordance with previous reports, the mutation rates of the KRAS gene in PDAs and undifferentiated carcinomas were high, up to $90-100 \%(40,41)$. Mutations in the KRAS gene have been identified in $36-81 \%$ of IPMN. In the present study, the mutation rate of the KRAS gene was $50 \%$ in IPMN and 54\% in IPMN-associated carcinomas, significantly lower compared with PDAs and PanINs. This result suggested that IPMN may employ a different carcinogenic pathway compared with the PanIN-PDA sequence $(11,14,42,43)$. The KRAS mutation foci of IPMN were primarily G12D and G12V, similar to PDAs (22,42-45). Wu et al (22) identified a high prevalence of KRAS mutations at codon 12 . In accordance with the present results, the most commonly altered amino acid was G12D and G12V. The other amino acid alterations, including G12S, G12A, G12C and G12R, occurred at a low rate. No mutations were detected in codon 13 of exon 2 in the present study. Nikiforova et al (45) reported a 4\% mutation rate in codon 13 of pancreatic cysts (G13D); it may be considered that the small number of cases and the Chinese cohort used in the present study may account for this difference. In addition, previous studies have demonstrated that Chinese cohorts have a lower KRAS mutation rate compared with European or Japanese cohorts $(46,47)$.

In the present study, the rate of mutation in IPMN with low-grade dysplasia was 58\%, which suggested that KRAS mutation is a very early event during tumorigenesis and serves an important role in the development of IPMNs, similar to what has been observed in PanIN (47). KRAS gene mutations have been demonstrated to be increased in proportion to the degree of dysplasia, and were previously identifies in 36, 44 and $87 \%$ of PanIN1A, PanIN1B and PanIN-2/3 lesions, respectively (48). KRAS mutation has been observed to serve an important role in the PanIN-ductal adenocarcinoma sequence of the pancreas $(49,50)$. Regarding the association between KRAS mutations and subtypes of IPMN, the previous studies in the literature are controversial (10-14,19-21). A particular study reported that KRAS mutation was not associated with classification or histological subtype (43), while another study suggested that KRAS mutation correlated with malignancy in IPMN (51). The present results were similar to those of Wu et al (22) and Nikiforova et al (45), demonstrating that higher KRAS mutation rates were identified in low- and high-grade IPMN compared with intermediate-grade IPMN. This suggested that KRAS mutations were early onset and had no association with the degree of dysplasia in IPMN.

KRAS mutations were present in all histological subtypes except oncocytic IPMN. Wu et al (22) demonstrated that KRAS mutations had the highest frequency in pancreatobiliary-type IPMN and the lowest frequency in intestinal-type IPMN. Nikiforova et al (45) reported that gastric-type IPMN had the highest mutation prevalence. The results of the present study demonstrated that low-grade IPMN and gastric-type IPMN tended to have KRAS mutations, although it was not possible to acquire statistical data due to the low case numbers. Typically, intestinal-type IPMN had the lowest KRAS mutant prevalence. This may explain why IPMN with intermediate-grade dysplasia had the lowest mutation prevalence. However, no KRAS mutations were detected in the three oncocytic-type IPMN cases, consistent with other reports in the literature (52). KRAS mutation prevalence was markedly low in intestinal-type IPMN with low- and intermediate-grade dysplasia, and high in gastric-type IPMN. Almost no low- or intermediate-grade dysplasia was identified in pancreatobiliary type IPMN, while this type of dysplasia was frequently identified in intestinal and gastric type IPMN. KRAS mutation frequency differed between gastric-, intestinal- and pancreatobiliary type of IPMN and therefore the overall KRAS mutation rate depended on the relative proportion of each type of IPMN in the studied cohort. The higher the proportion of gastric type IPMN in the studied cohort, the higher the frequency of KRAS mutation. In a similar way, KRAS mutation prevalence in IPMN-associated carcinoma was determined to a great extent by the ratio of intestinal- and pancreatobiliary-type IPMN. The heterogeneity of IPMN may explain the marked difference in various studies into KRAS mutation prevalence.

In the present study, intestinal type IPMN was the only type with low-grade to high-grade dysplasia and infiltrating adenocarcinoma. There was no gastric-type associated adenocarcinoma, or pancreatobiliary-type with low- and intermediate dysplasia. Therefore, intestinal type IPMN was selected to study the association between KRAS mutation and dysplasia. It was demonstrated that the frequency of KRAS mutation increased with the increase of the degree of dysplasia and invasion. Intestinal-type IPMN-associated carcinomas exhibited higher KRAS mutation frequencies compared with non-invasive IPMN.

Previously, a number of studies have discussed the association between KRAS mutation and survival in patients with pancreatic carcinomas, and reported that KRAS mutations were not associated with poor prognosis in patients with PDA $(15,17,53)$, and a KRAS mutant allele-specific imbalance was associated with worse prognosis in pancreatic cancer and progression to undifferentiated carcinoma of the pancreas (9). From the data in the present study, it was suggested that there was no significant difference in KRAS mutation frequencies among the different histological subtypes, associated invasive carcinoma incidence, infiltrative depth, differentiation, lymph node metastasis or invasive scope.

The Kaplan-Meier survival curve demonstrated that the survival rate had no association with KRAS mutation. Therefore, the detection of KRAS mutations in a biopsy from a patient with IPMN may not be used to draw the conclusion that the lesion had high dysplasia, infiltration or a bad prognosis. KRAS mutations do not serve a role in predicting the prognosis or survival of patients with IPMN.

The present study elaborated upon the clinical significance of KRAS mutations. However, the present study had a relatively small sample size. A future study is required with a larger sample size. Furthermore, further studies may focus on other molecular alterations in addition to KRAS. 
In conclusion, KRAS mutation prevalence in intestinal-type IPMN was the lowest, and the incidence increases with the degree of dysplasia and invasion in intestinal-type IPMNs. KRAS mutation in IPMN does not correlate with histological subtype, dysplasia grade, invasion or survival.

\section{Acknowledgements}

Not applicable.

\section{Funding}

The present study was supported by the Foundation of Pathologic Research Centre of the China Academy of Medical Sciences (grant nos. 2015PT320002 and 2016ZX310176-3).

\section{Availability of data and materials}

The datasets used and/or analyzed during the current study are available from the corresponding author on reasonable request.

\section{Authors' contributions}

JC contributed to the conception of the idea. XC and YL contributed to the literature search and drafting of the manuscript. YW performed the molecular genetic studies. JW contributed to the statistical work and language editing. All authors critically reviewed and accepted the final version of the manuscript. JC will accept full responsibility for the present study.

\section{Ethics approval and consent to participate}

This study was approved by Ethics Committee of Peking Union Medical College Hospital and informed consents were obtained from all patients.

\section{Consent for publication}

Not applicable.

\section{Competing interests}

The authors declare that they have no competing interests.

\section{References}

1. Fernández-del Castillo C and Adsay NV: Intraductal papillary mucinous neoplasms of the pancreas. Gastroenterology 139 708-713, e1-2, 2010

2. Adsay NV, Conlon KC, Zee SY, Brennan MF and Klimstra DS: Intraductal papillary-mucinous neoplasms of the pancreas: An analysis of in situ and invasive carcinomas in 28 patients. Cancer 94: 62-77, 2002

3. Fernández-del Castillo C, Targarona J, Thayer SP, Rattner DW, Brugge WR and Warshaw AL: Incidental pancreatic cysts: Clinicopathologic characteristics and comparison with symptomatic patients. Arch Surg 138: 427-434, 2003.

4. Andrejevic-Blant S, Kosmahl M, Sipos B and Klöppel G: Pancreatic intraductal papillary-mucinous neoplasms: A new and evolving entity. Virchows Arch 451: 863-869, 2007.

5. Kosmahl M, Pauser U, Peters K, Sipos B, Lüttges J, Kremer B and Klöppel G: Cystic neoplasms of the pancreas and tumor-like lesions with cystic features: A review of 418 cases and a classification proposal. Virchows Arch 445: 168-178, 2004.
6. Balzano G, Zerbi A and Di Carlo V: Intraductal papillary mucinous tumors of the pancreas: Incidence, clinical findings and natural history. JOP 6 (1 Suppl): S108-S111, 2005.

7. Hruban RH and Klimsra DS: Intraductal neoplasms. In: Hruban RH, Pitman MB, Klimsstra DS (eds) AFIP Atlas of Tumor Pathology, Series 4: Tumors of the Pancreas, 2007.

8. Nara S, Shimada K, Kosuge T, Kanai Y and Hiraoka N: Minimally invasive intraductal papillary-mucinous carcinoma of the pancreas: Clinicopathologic study of 104 intraductal papillary-mucinous neoplasms. Am J Surg Pathol 32: 243-255, 2008.

9. Krasinskas AM, Moser AJ, Saka B, Adsay NV and Chiosea SI: KRAS mutant allele-specific imbalance is associated with worse prognosis in pancreatic cancer and progression to undifferentiated carcinoma of the pancreas. Mod Pathol 26: 1346-1354, 2013

10. Sessa F, Solcia E, Capella C, Bonato M, Scarpa A, Zamboni G, Pellegata NS, Ranzani GN, Rickaert F and Klöppel G: Intraductal papillary-mucinous tumours represent a distinct group of pancreatic neoplasms: An investigation of tumour cell differentiation and K-ras, p53 and c-erbB-2 abnormalities in 26 patients. Virchows Arch 425: 357-367, 1994.

11. Tada M, Omata M and Ohto M: Ras gene mutations in intraductal papillary neoplasms of the pancreas. Analysis in five cases. Cancer 67: 634-637, 1991.

12. Satoh K, Shimosegawa T, Moriizumi S, Koizumi M and Toyota T: K-ras mutation and 553 protein accumulation in intraductal mucin-hypersecreting neoplasms of the pancreas. Pancreas 12: 362-368, 1996.

13. Satoh K, Sawai T, Shimosegawa T, Koizumi M, Yamazaki T, Mochizuki F and Toyota T: The point mutation of c-Ki-ras at codon 12 in carcinoma of the pancreatic head region and in intraductal mucin-hypersecreting neoplasm of the pancreas. Int J Pancreatol 14: 135-143, 1993.

14. Uemura K, Hiyama E, Murakami Y, Kanehiro T, Ohge H, Sueda T and Yokoyama T: Comparative analysis of K-ras point mutation, telomerase activity, and $\mathrm{p} 53$ overexpression in pancreatic tumours. Oncol Rep 10: 277-283, 2003.

15. Oliveira-Cunha M, Hadfield KD, Siriwardena AK and Newman W: EGFR and KRAS mutational analysis and their correlation to survival in pancreatic and periampullary cancer. Pancreas 41: 428-434, 2012.

16. Kim ST, Lim DH, Jang KT, Lim T, Lee J, Choi YL, Jang HL, Yi JH, Baek KK, Park SH, et al: Impact of KRAS mutations on clinical outcomes in pancreatic cancer patients treated with first-line gemcitabine-based chemotherapy. Mol Cancer Ther 10: 1993-1999, 2011.

17. Schultz NA, Roslind A, Christensen IJ, Horn T, Høgdall E, Pedersen LN, Kruhøffer M, Burcharth F, Wøjdemann M and Johansen JS: Frequencies and prognostic role of KRAS and BRAF mutations in patients with localized pancreatic and ampullary adenocarcinomas. Pancreas 41: 759-766, 2012.

18. Bournet B, Muscari F, Guimbaud R, Cordelier P and Buscail L: KRAS mutations and their correlation with survival of patients with advanced pancreatic cancer. Pancreas 42: 543-544, 2013.

19. Z'Graggen K, Rivera JA, Compton CC, Pins M, Werner J, Fernández-del Castillo C, Rattner DW, Lewandrowski KB, Rustgi AK and Warshaw AL: Prevalence of activating K-ras mutations in the evolutionary stages of neoplasia in intraductal papillary mucinous tumors of the pancreas. Ann Surg 226: 491-500, 1997.

20. Schönleben F, Qiu W, Ciau NT, Ho DJ, Li X, Allendorf JD, Remotti HE and Su GH: PIK3CA mutations in intraductal papillary mucinous neoplasm/carcinoma of the pancreas. Clin Cancer Res 12: 3851-3855, 2006.

21. Yoshizawa K, Nagai H, Sakurai S, Hironaka M, Morinaga S, Saitoh K and Fukayama M: Clonality and K-ras mutation analyses of epithelia in intraductal papillary mucinous tumor and mucinous cystic tumor of the pancreas. Virchows Arch 441: 437-443, 2002.

22. Wu J,MatthaeiH, Maitra A, Dal Molin M, WoodLD, Eshleman JR, Goggins M, Canto MI, Schulick RD, Edil BH, et al: Recurrent GNAS mutations define an unexpected pathway for pancreatic cyst development. Sci Transl Med 3: 92ra66, 2011.

23. Furukawa T, Kuboki Y, Tanji E, Yoshida S, Hatori T, Yamamoto M, Shibata N, Shimizu K, Kamatani N and Shiratori K: Whole-exome sequencing uncovers frequent GNAS mutations in intraductal papillary mucinous neoplasms of the pancreas. Sci Rep 1: 161, 2011. 
24. Swerdlow SH, Campo E and Harris NL (eds): WHO classification of tumors of the digestive system. 4th edition. IARC, pp304-313, 2010.

25. Bando H, Tsuchihara K, Yoshino T, Kojima M, Ogasawara N, Fukushima H, Ochiai A, Ohtsu A and Esumi H: Biased discordance of KRAS mutation detection in archived colorectal cancer specimens between the ARMS-Scorpion method and direct sequencing. Jpn J Clin Oncol 41: 239-244, 2011.

26. Franklin WA, Haney J, Sugita M, Bemis L, Jimeno A and Messersmith WA: KRAS mutation: Comparison of testing methods and tissue sampling techniques in colon cancer. $\mathrm{J} \mathrm{Mol}$ Diagn 12: 43-50, 2010.

27. Adsay NV, Merati K, Basturk O, Iacobuzio-Donahue C Levi E, Cheng JD, Sarkar FH, Hruban RH and Klimstra DS: Pathologically and biologically distinct types of epithelium in intraductal papillary mucinous neoplasms: Delineation of an 'intestinal' pathway of carcinogenesis in the pancreas. Am J Surg Pathol 28: 839-848, 2004

28. Hruban RH, Takaori K, Klimstra DS, Adsay NV, Albores-Saavedra J, Biankin AV, Biankin SA, Compton C, Fukushima N, Furukawa T, et al: An illustrated consensus on the classification of pancreatic intraepithelial neoplasia and intraductal papillary mucinous neoplasms. Am J Surg Pathol 28 : 977-987, 2004

29. Lüttges J, Zamboni G, Longnecker D and Klöppel G: The immunohistochemical mucin expression pattern distinguishes different types of intraductal papillary mucinous neoplasms of the pancreas and determines their relationship to mucinous noncystic carcinoma and ductal adenocarcinoma. Am J Surg Pathol 25: 942-948, 2001

30. Furukawa T, Klöppel G, Volkan Adsay N, Albores-Saavedra J, Fukushima N, Horii A, Hruban RH, Kato Y, Klimstra DS Longnecker DS, et al: Classification of types of intraductal papillary-mucinous neoplasm of the pancreas: A consensus study. Virchows Arch 447: 794-799, 2005.

31. Nakamura A, Horinouchi M, Goto M, Nagata K, Sakoda K, Takao S, Imai K, Kim YS, Sato E and Yonezawa S: New classification of pancreatic intraductal papillary-mucinous tumour by mucin expression: Its relationship with potential for malignancy. J Pathol 197: 201-210, 2002.

32. Tanaka M, Chari S, Adsay V, Fernandez-del Castillo C, Falconi M, Shimizu M, Yamaguchi K, Yamao K and Matsuno S International Association of Pancreatology: International consensus guidelines for management of intraductal papillary mucinous neoplasms and mucinous cystic neoplasms of the pancreas. Pancreatology 6: 17-32, 2006.

33. Mino-Kenudson M, Fernández-del Castillo $\mathrm{C}$, Baba $\mathrm{Y}$, Valsangkar NP, Liss AS, Hsu M, Correa-Gallego C, Ingkakul T, Perez Johnston R, Turner BG, et al: Prognosis of invasive intraductal papillary mucinous neoplasm depends on histological and precursor epithelial subtypes. Gut 60: 1712-1720, 2011.

34. Furukawa T, Hatori T, Fujita I, Yamamoto M, Kobayashi M, Ohike N, Morohoshi T, Egawa S, Unno M, Takao S, et al: Prognostic relevance of morphological types of intraductal papillary mucinous neoplasms of the pancreas. Gut 60: 509-516, 2011.

35. Kang MJ, Lee KB, Jang JY, Han IW and Kim SW: Evaluation of clinical meaning of histological subtypes of intraductal papillary mucinous neoplasm of the pancreas. Pancreas 42: 959-966, 2013

36. Poultsides GA, Reddy S, Cameron JL, Hruban RH, Pawlik TM, Ahuja N, Jain A, Edil BH, Iacobuzio-Donahue CA, Schulick RD and Wolfgang CL: Histopathologic basis for the favorable survival after resection of intraductal papillary mucinous neoplasm-associated invasive adenocarcinoma of the pancreas. Ann Surg 251: 470-476, 2010.

37. Adsay NV, Pierson C, Sarkar F, Abrams J, Weaver D, Conlon KC Brennan MF and Klimstra DS: Colloid (mucinous noncystic) carcinoma of the pancreas. Am J Surg Pathol 25: 26-42, 2001.

38. Sadakari Y, Ohuchida K, Nakata K, Ohtsuka T, Aishima S, Takahata S, Nakamura M, Mizumoto K and Tanaka M: Invasive carcinoma derived from the nonintestinal type intraductal papillary mucinous neoplasm of the pancreas has a poorer prognosis than that derived from the intestinal type. Surgery 147: 812-817, 2010 .
39. Takasu N, Kimura W, Moriya T, Hirai I, Takeshita A, Kamio Y and Nomura T: Intraductal papillary-mucinous neoplasms of the gastric and intestinal types may have less malignant potential than the pancreatobiliary type. Pancreas 39: 604-610, 2010.

40. Moskaluk CA, Hruban RH and Kern SE: p16 and K-ras gene mutations in the intraductal precursors of human pancreatic adenocarcinoma. Cancer Res 57: 2140-2143, 1997.

41. Rozenblum E, Schutte M, Goggins M, Hahn SA, Panzer S, Zahurak M, Goodman SN, Sohn TA, Hruban RH, Yeo CJ and Kern SE: Tumor-suppressive pathways in pancreatic carcinoma. Cancer Res 57: 1731-1734, 1997.

42. Schonleben F, Qiu W, Remotti HE, Hohenberger W and Su GH: PIK3CA, KRAS, and BRAF mutations in intraductal papillary mucinous neoplasm/carcinoma (IPMN/C) of the pancreas. Langenbecks Arch Surg 393: 289-296, 2008.

43. Chadwick B, Willmore-Payne C, Tripp S, Layfield LJ, Hirschowitz S and Holden J: Histologic, immunohistochemical, and molecular classification of 52 IPMNs of the pancreas. Appl Immunohistochem Mol Morphol 17: 31-39, 2009.

44. Izeradjene K, Combs C, Best M, Gopinathan A, Wagner A, Grady WM, Deng CX, Hruban RH, Adsay NV, Tuveson DA and Hingorani SR: Kras(G12D) and Smad4/Dpc4 haploinsufficiency cooperate to induce mucinous cystic neoplasms and invasive adenocarcinoma of the pancreas. Cancer Cell 11: 229-243, 2007.

45. Nikiforova MN, Khalid A, Fasanella KE, McGrath KM, Brand RE, Chennat JS, Slivka A, Zeh HJ, Zureikat AH, Krasinskas AM, et al: Integration of KRAS testing in the diagnosis of pancreatic cystic lesions: A clinical experience of 618 pancreatic cysts. Mod Pathol 26: 1478-1487, 2013.

46. Kwon MJ, Jeon JY, Park HR, Nam ES, Cho SJ, Shin HS, Kwon JH, Kim JS, Han B, Kim DH and Choi YL: Low frequency of KRAS mutation in pancreatic ductal adenocarcinomas in Korean patients and its prognostic value. Pancreas 44: 484-492, 2015.

47. Kanda M, Matthaei H, Wu J, Hong SM, Yu J, Borges M, Hruban RH, Maitra A, Kinzler K, Vogelstein B and Goggins M: Presence of somatic mutations in most early-stage pancreatic intraepithelial neoplasia. Gastroenterology 142: 730-733 e9, 2012.

48. Löhr M, Klöppel G, Maisonneuve P, Lowenfels AB and Lüttges J: Frequency of K-ras mutations in pancreatic intraductal neoplasia associated with pancreatic ductal adenocarcinoma and chronic pancreatitis: A meta-analysis. Neoplasia 7: 17-23, 2005.

49. Shi C, Hong SM, Lim P, Kamiyama H, Khan M, Anders RA, Goggins M, Hruban RH and Eshleman JR: KRAS2 mutations in human pancreatic acinar-ductal metaplastic lesions are limited to those with PanIN: Implications for the human pancreatic cancer cell of origin. Mol Cancer Res 7: 230-236, 2009.

50. di Magliano MP and Logsdon CD: Roles for KRAS in pancreatic tumor development and progression. Gastroenterology 144: 1220-1229, 2013.

51. Jang JY, Park YC, Song YS, Lee SE, Hwang DW, Lim CS, Lee HE, Kim WH and Kim SW: Increased K-ras mutation and expression of S100A4 and MUC2 protein in the malignant intraductal papillary mucinous tumor of the pancreas. J Hepatobiliary Pancreat Surg 16: 668-674, 2009.

52. Chung SM, Hruban RH, Iacobuzio-Donahue CA, Adsay NV, Zee SY and Klimstra DS: Analysis of molecular alterations and differentiation pathways in intraductal oncocytic papillary neoplasm of the pancreas. Mod Pathol 18: 277A-288A, 2005.

53. Kim J, Jang KT, Mo Park S, Lim SW, Kim JH, Lee KH, Lee JK, Heo JS, Choi SH, Choi DW, et al: Prognostic relevance of pathologic subtypes and minimal invasion in intraductal papillary mucinous neoplasms of the pancreas. Tumour Biol 32: 535-542, 2011.

This work is licensed under a Creative Commons Attribution 4.0 International (CC BY-NC 4.0) License 